\title{
Parallel Processing of Proprioceptive Signals by Spiking Local Interneurons and Motor Neurons in the Locust
}

\author{
M. Burrows \\ Department of Zoology, University of Cambridge, Cambridge CB2 3EJ, England
}

The connections made by afferents from a proprioceptor at the femorotibial joint in a hind leg of a locust, the femoral chordotonal organ (FCO), were determined by making intracellular recordings from motor neurons and spiking local interneurons in the central nervous system and from afferent cell bodies in the periphery. Staining the central projections of the afferent neurons with dye introduced into their axons at the receptor, and the intracellular injection of dye into motor neurons and interneurons, shows that the branches of all 3 types of neuron overlap in specific regions of neuropile. Afferents excited by a movement of the receptor apodeme that is equivalent to an imposed extension of the femorotibial joint excite flexor tibiae motor neurons and some spiking local interneurons with cell bodies at the ventral midline of the metathoracic ganglion. The opposite movement excites extensor tibiae motor neurons and a different set of spiking local interneurons. Spikes in afferents that excite flexor motor neurons evoke depolarizing potentials that follow each spike with a consistent central latency of approximately $\mathbf{1 . 5}$ msec. The amplitude of the depolarizing potentials is dependent upon the membrane potential of the motor neuron. This evidence points to the connection being direct and to the potentials' being EPSPs. Simultaneous recordings from certain spiking local interneurons and certain flexor motor neurons show that they receive many synaptic potentials in common and are driven in a parallel fashion by movements of the receptor apodeme. Spikes of some afferents evoke EPSPs in both neurons with the same consistency and latency. An afferent can therefore synapse directly upon a motor neuron and a spiking local interneuron. Each afferent synapses on several motor neurons and possibly upon several interneurons. In turn, each motor neuron and each interneuron receives inputs from several afferents.

During locomotion, a limb must subserve the dual functions of moving the body and bearing its load. Proprioceptors monitor both the movement of the joints and the current mechanical status of the muscle, therefore providing the means for rapid corrections of the motor outflow. Adjustments to posture and

\footnotetext{
Received May 20, 1986; accepted Oct. 2, 1986.

This work was supported by NIH Grant NS 16058. I thank A. Watson and K. Seymour for responding to the challenge to stain the central projections of the FCO afferents, G. Laurent for the drawing in Figure 1, and B. Watkins for the electron microscopy. The constructive comments of my colleagues in Cambridge and Konstanz on this manuscript are gratefully acknowledged.

Correspondence should be addressed to Dr. M. Burrows, Department of Zoology, University of Cambridge, Downing Street, Cambridge CB2 3EJ, England. Copyright (C) 1987 Society for Neuroscience $0270-6474 / 87 / 041064-17 \$ 02.00 / 0$
}

locomotion are initiated at several levels in the nervous system so that the central effects of the proprioceptive afferents will be widespread. 1 a afferents from the muscle spindles of vertebrates mediate stretch reflexes by synapsing directly on agonist motor neurons and, at the same time, on la inhibitory interneurons to inhibit antagonists (for reviews, see Matthews, 1972; Brown, 1981). By contrast, $1 \mathrm{~b}$ afferents from tendon organs limit tension via pathways involving interneurons.

In arthropods, similar constraints on movement have led to similar control mechanisms, involving a variety of proprioceptors. In crayfish, afferents from muscle receptor organs that are excited when the abdomen is flexed synapse upon extensor motor neurons (Wine, 1977). In crabs, nonspiking afferents from a receptor excited when a leg is remoted synapse directly on promotor motor neurons (Blight and Llinás, 1980). In locusts, a stretch receptor excited when a wing is elevated synapses on wing depressor motor neurons (Burrows, 1975) and on interneurons (Pearson et al., 1983). In cockroaches, afferents from a hair plate on the trochanter, excited when the leg is levated, synapse directly on depressor motor neurons (Pearson et al., 1976).

Analyzing any of these reflex pathways in detail requires an accessible proprioceptor. An extensively studied invertebrate proprioceptor is the femoral chordotonal organ of insects, but, surprisingly, nothing is known of the central connections made by its afferents. This sense organ consists of a group of receptor cells embedded in connective tissue and linked by a stiff apodeme to an insertion point close to that of the extensor tibiae muscle (Usherwood et al., 1968). The receptors code the position of the femorotibial joint, the direction and velocity of its movement with range fractionation, as well as the force generated by the extensor muscle (Hofmann et al., 1985; Zill, 1985a). There is no direct efferent control. A particularly attractive feature of this proprioceptor is that its apodeme can be moved experimentally so that its effects on leg movements can be studied in open-or closed-loop conditions, without interference from other sensory signals. In this way, its essential part in the control of the joint has been extensively analyzed in stick insects (Bässler, 1983). It mediates resistance reflexes in muscles that move the femorotibial joint (Usherwood et al., 1968; Burns, 1974; Bässler, 1977; Field and Burrows, 1982), an intersegmental reflex (Field and Rind, 1981), and has been suggested to play a role in triggering the jump of a locust (Bässler, 1968; Pearson et al., 1980). Nevertheless, the reflex responses are not rigid (Field and Burrows, 1982) and may show phase reversals (Bässler, 1976) or change during voluntary movements so that, for example, flexor motor neurons are excited by both extension and flexion of the joint (Zill, 1985b). These results suggest con- 
flicting requirements of the connections made by the afferents in the central nervous system: fast reliable pathways that must be modifiable to fit the current behavior of the animal.

In this paper I examine 2 possible central targets for these proprioceptive afferents, leg motor neurons and spiking local interneurons. Consideration of the motor neurons is restricted to those that innervate muscles moving the femorotibial joint and the spiking local interneurons which belong to a ventral midline group (Siegler and Burrows, 1984). These receive direct inputs from hairs and campaniform sensilla (Siegler and Burrows, 1983) and map the surface of a leg as a series of overlapping receptive fields (Burrows and Siegler, 1985). Some also respond in a variety of ways to movements of the joints of a leg (Burrows, 1985). I show here that the afferents from the femoral chordotonal organ synapse on motor neurons that control movements of the femorotibial joint and, in parallel, on certain of these spiking local interneurons.

\section{Materials and Methods}

Adult male and female locusts, Schistocerca gregaria (Forskal), from our crowded laboratory culture were mounted ventral surface uppermost. The femur of the left hind leg was rotated so that the anterior surface was also uppermost, and it was then firmly embedded in plasticine. The tibia and tarsus of this and all the other legs, as well as the head and abdomen, were free to move. The meso- and metathoracic ganglia were exposed and stabilized on a wax-coated steel platform and the thoracic cavity was perfused with a constant flow of saline. The sheath of the ganglion was treated with a $1 \%(\mathrm{wt} / \mathrm{vol})$ solution of protease (Sigma type XIV) before recording began. The cell bodies of motor neurons and interneurons were impaled with microelectrodes filled with $2 \mathrm{M}$ potassium acetate having DC resistances of 50-80 M 2 . Motor neurons were identified by correlating their intracellularly recorded spikes with potentials recorded extracellularly from a particular muscle, using a pair of $50 \mu \mathrm{m}$ steel wires insulated except for their tips. In addition, flexor tibiae motor neurons could be recognized by the occurrence of a large depolarizing potential whenever an antidromic spike was induced in the fast extensor tibiae motor neuron (see Hoyle and Burrows, 1973). Interneurons were characterized as members of the midline group of spiking local interneurons by the position of their cell bodies and by plotting their receptive fields on the left hind leg following mechanical stimulation of proprioceptors and exteroreceptors. Some interneurons were injected with cobalt (Pitman et al., 1972) and could be confirmed as being spiking local interneurons of the midline group by their morphology (see Siegler and Burrows, 1984; Burrows, 1985 for details).

The apodeme of the femoral chordotonal organ (FCO) was exposed by dissecting away cuticle on the anterior face of the femur proximal to the heavily sclerotized and blackened semilunar process (Fig. 1). The femorotibial joint was set at $80^{\circ}$, and the apodeme grasped between the tips of forceps attached to a vibrator (Ling-Altec type 101). The apodeme was then cut distally, so that movement of the proximal part in waveforms of controlled frequencies and amplitudes would excite receptors of the FCO. A $500 \mu \mathrm{m}$ movement of the apodeme corresponds to a $40^{\circ}$ movement of the femorotibial joint. There is a linear relationship between movement of the apodeme and joint angle between $20^{\circ}$ and $130^{\circ}$ (Field and Burrows, 1982). A stretch applied to the apodeme corresponds to the movement that would normally occur during a flexion of the femorotibial joint and, similarly, a movement that allows the FCO to relax corresponds to an extension of the joint. A movement of the apodeme will be described as either a flexion or an extension, reflecting the way it is normally activated in the leg. The tibia was free to move, so that the flexor strand of the FCO (see Field and Burrows, 1982) and its associated receptor cell (Bräunig, 1985) could be activated independently by imposed or active movements of the tibia.

Spikes of the FCO receptors were recorded extracellularly by a pair of hook electrodes, made from $100-\mu$ m-diameter silver wire, and placed on N5b1 just proximal to the point at which the FCO nerve joins. Nerve $5 \mathrm{~b} 1$ continues into the tibia to supply a group of campaniform sensilla and the subgenual organ (Heitler and Burrows, 1977). Input from these tibial receptors was abolished in some experiments by severing the nerve distal to the point at which the FCO nerve joins. Individual sensory cell bodies were impaled by a microelectrode. The receptor was not pretreated with protease. To achieve the necessary stability, a waxcovered platform, similar to but smaller than that used for the ganglia, was slipped beneath the FCO to isolate it from movements caused by contractions of the muscles or pulsations of the tracheae. The apodeme was held in a fixed position by the forceps. Receptor cells thus could not be excited by movement, but the one that was impaled could be induced to spike by the injection of current through the recording electrode.

All recordings were stored on magnetic tape for later display on a Gould ES1000 recorder or, after capture on a digital storage oscilloscope, on an $X-Y$ plotter. The physiological results are based on 76 successful simultaneous recordings from an interneuron and a motor neuron in 33 locusts.

The number of cell bodies of sensory afferents in the FCO, and the projections of their axons into the metathoracic ganglion were revealed by staining with cobalt. The nerve leading from the FCO before it joins $\mathrm{N} 5 \mathrm{~b} 1$ was exposed, and both distal and proximal cut ends placed in a $2 \%$ solution of cobalt chloride. At this point, the nerve also contained afferents from some femoral hairs. It is inevitable, using this method, that some of the central projections of these neurons will also be stained, and that some of the smaller-diameter axons of the FCO may fail to stain. The cobalt was allowed to diffuse for 2-6 d before it was precipitated as the sulfide. A dehydrated and cleared $\mathrm{FCO}$ was then viewed as a whole-mount, while the central projections in the metathoracic ganglion were intensified with silver (Bacon and Altman, 1977). Eight successful and consistent stains of the peripheral cell bodies, and 5 of the central axonal projections were obtained.

The number of axons in the nerve just as it enters the FCO was revealed by electron microscopy in 2 locusts. The nerve, attached at one end to the FCO, and at the other to N5bl, was fixed in a $2.5 \%$ glutaraldehyde solution in $0.05 \mathrm{~m}$ phosphate buffer, osmicated, and examined in a Philips 200 electron microscope.

\section{Results}

\section{The femoral chordotonal organ}

The femoral chordotonal organ consists of a group of sensory cells, suspended by ligaments, that attach to the anterior face of the femur by an apodeme and elastic strand (Fig. $1 A$ ). The stiff apodeme, into which the dendrites of the receptor cells project, runs distally, inserting on a tibial protuberance lateral to the insertion of the tendon of the extensor tibiae muscle. The elastic flexor strand attaches close to the insertion of the flexor tibiae muscle and is associated with a single receptor cell (Bräunig, 1985) that is not studied here. The axons of the sensory neurons form a bundle that, after some $500 \mu \mathrm{m}$, joins nerve $5 \mathrm{~b} 1$. Sections of this nerve bundle from 2 locusts, viewed in the electron microscope, reveal the profiles of 37 axons in each. The diameters range from 1 to $6 \mu \mathrm{m}$, with most between 2.5 and 5 $\mu \mathrm{m}$. Allowing cobalt to diffuse into the cut ends of these axons towards the chordotonal organs reveals the presence of an average of 38 cell bodies (range, 29-44; $n=8$ ) (Fig. $1 B$ ). The largest cell is $70 \mu \mathrm{m}$ and the smallest $15 \mu \mathrm{m}$ in diameter, but the majority are between 20 and $30 \mu \mathrm{m}$.

\section{Central projections of FCO afferents}

If afferents from the FCO are to synapse directly on leg motor neurons and spiking local interneurons, then all 3 classes of neuron must project to the same region or regions of neuropile. Afferents from the FCO project only to the ipsilateral half of the metathoracic ganglion (Fig. $2 A$ ). The projection consists of a prominent region of lateral branching and a more medial area from which 2 small bundles of fibers run toward the midline. The most anterior of these bundles runs between the ventral intermediate (VIT) and ventral lateral (VLT) longitudinal tracts to join dorsal commissure III (DCIII) (Fig. 3A). The more posterior bundle runs between the ventral and dorsal intermediate tracts (VIT and DIT) to join dorsal commissure IV (DCIV) (Fig. 


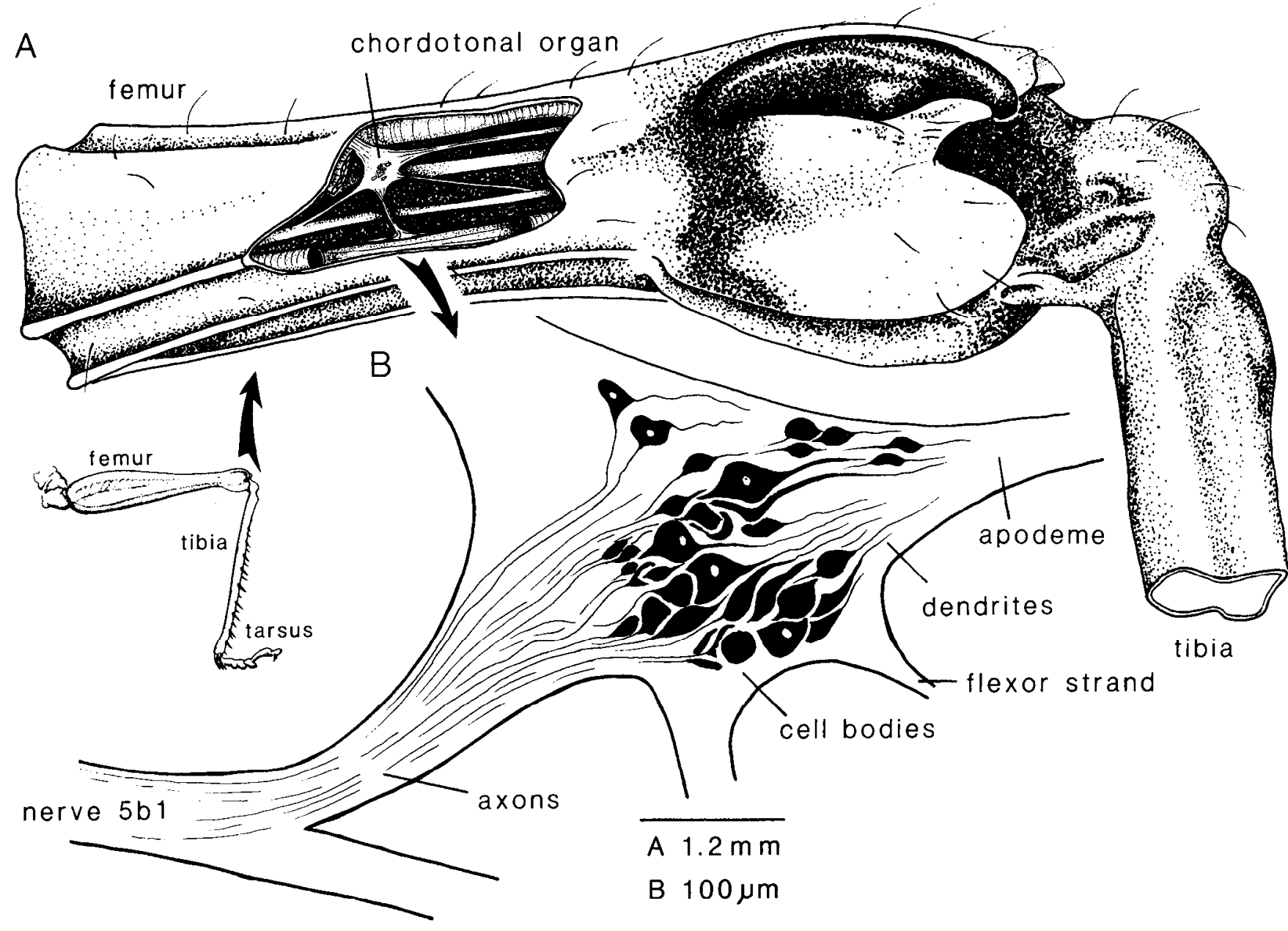

Figure 1. Femoral chordotonal organ of a left hind leg. A, Drawing of the femorotibial joint with a small window opened in the cuticle of the anterior surface of the femur to reveal the chordotonal organ. A small bundle of fibers of the flexor tibiae muscle, which lies anterior to the organ, and of the ventral trachea are cut. $B$, Chordotonal organ in more detail to show its sensory neurons after staining with cobalt introduced into nerve 5b1. The dendrites extend into the apodeme, which then inserts close to the tendon of the extensor tibiae muscle. Scale bar: $A$, 1.2 mm; $B$, $100 \mu \mathrm{m}$.

$3 B$ ). The main body of branches lies at the level of the VIT and the median ventral tract (MVT) (Fig. 3,B,C). The lateral branches are close to the lateral boundary of the neuropile, and the most posterior branches are also the most dorsal (Fig. $3 D$ ). Branches are conspicuously absent from the most ventral regions of neuropile. The overall projection is similar to that described for the tibial afferents whose axons run in N5bl (Grosch et al., 1985). In the stains here, leakage into these adjacent axons is absent, though the possibility that some femoral hair afferents are also stained cannot be excluded. The tibial afferents, therefore, either have a similar pattern of projections to the FCO, or the stains from Grosch et al. (1985) include the FCO afferents.

Individual FCO afferents may have different patterns of central branches (Fig. 2,B-E). Some have medial branches running toward DCIII or DCIV but not crossing the midline; some have only lateral branches; while others have both medial and lateral branches. It is not yet known whether these different anatomical types of projection correspond to neurons with particular types of physiological responses.

Flexor tibiae motor neurons that are excited by movements of the FCO apodeme have 2 regions with branches that overlap with the branches of the afferents (Fig. 4). First, branches extend medially from the primary neurite as it turns to run posteriorly and pass between DIT and VIT toward DCIV (Fig. $4, A, B$ ). This is thus a projection comparable to the more posterior of the 2 bundles of afferent fibers projecting toward the midline (Figs. $2 A ; 3 B$ ). Second, many branches run laterally from the more posterior region of the primary neurite and end in neuropile at the lateral boundary of the ganglion level with DIT and VIT. This projection is comparable to the lateral branches of the afferents (Figs. $2 A ; 3 C$ ).

Spiking local interneurons that are excited by movements of the FCO apodeme have 2 fields of branches (Fig. 5). The ventral field (Fig. $5 \mathrm{~A}$ ) is sparse in comparison with interneurons in the same population that receive inputs from exteroreceptors (Burrows and Siegler, 1984), and there are no branches in the most ventral regions of neuropile, where the hair afferents terminate (Fig. $5 B$ ). The dorsal field is more extensive and is linked to the ventral field by a single process (Fig. $5 A$ ). Overlap between the branches of this interneuron and the afferents could occur either in a region just lateral to VIT at an anteroposterior level marked by DCIII (Figs. $3 A ; 5 B$ ) or in more lateral and posterior regions of neuropile (Figs. 3,C,D; $5 C$ ).

\section{Effects on interneurons and motor neurons}

Movements of the apodeme of the chordotonal organ produce profound effects on the membrane potentials of motor neurons innervating femoral muscles and of some spiking local inter- 

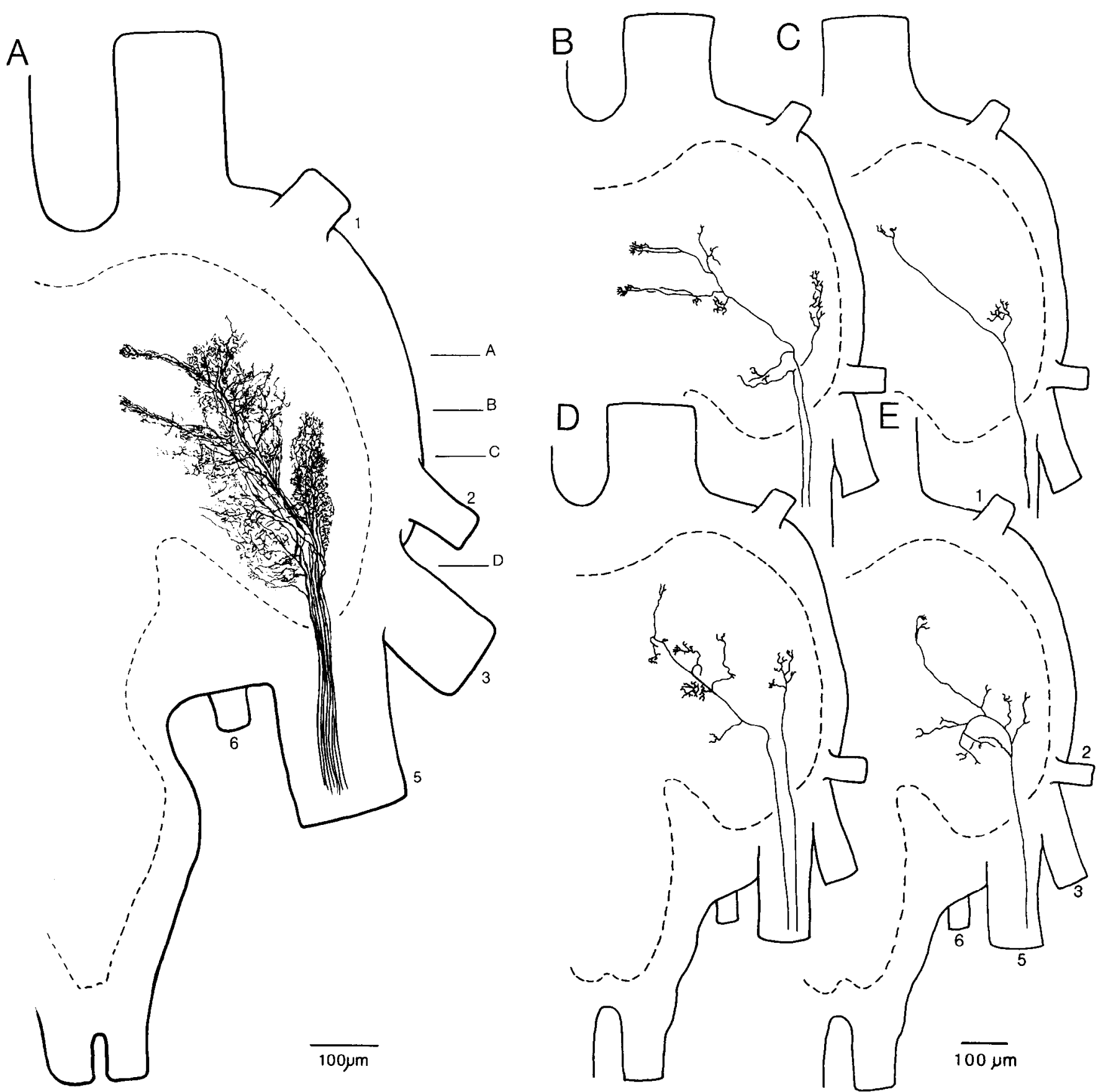

Figure 2. The projections of afferent fibers from the femoral chordotonal organ into the metathoracic ganglion. Cobalt was introduced into the cut end of the chordotonal nerve before it joined nerve $5 \mathrm{bl}$. $A$, Projection of many afferents from the FCO nerve. There is a prominent arborization in lateral neuropile, and 2 small bundles of fibers project toward the midline. Horizontal lines, $A-D$, show the levels of the transverse sections drawn in Figure 3. $B-E$, Projections of 6 afferent fibers that could be recognized in 1 stain of the chordotonal nerve in another locust. Lateral nerves $1-3,5$, and 6 are shown; other nerves are omitted. The dashed line indicates the boundary of the neuropile. The right half of the ganglion is viewed dorsally with anterior at the top.

neurons in the midline group. Two examples are shown in Figure 6. In the first, a movement of the apodeme corresponding to a flexion of the femorotibial joint depolarizes the slow extensor tibiae motor neuron and makes it spike (Fig. 6A). At the same time, a spiking local interneuron is hyperpolarized and its tonic sequence of spikes interrupted (Fig. $6 A$ ). On the opposite phase of the movement that corresponds to extension, the extensor motor neuron is hyperpolarized and the interneuron is depolarized and spikes rapidly. Flexor tibiae motor neurons must also be excited, because their spikes are recorded in the flexor muscle (Fig. $6 A$ ). These reciprocal effects in the 2 impaled neu- rons occur consistently with each repetition of the movement. In the second example, a flexor tibiae motor neuron is hyperpolarized by a flexion movement and depolarized by an extension (Fig. 6B). A different spiking local interneuron shows a reciprocal action; it spikes during a flexion and is inhibited by an extension movement (Fig. 6B). A simultaneous recording from the FCO nerve shows that afferents spike during both phases of the movement.

These effects on the motor neurons constitute a resistance reflex, whereby an imposed flexion of the joint would be met by increased activity of extensor motor neurons and an imposed 

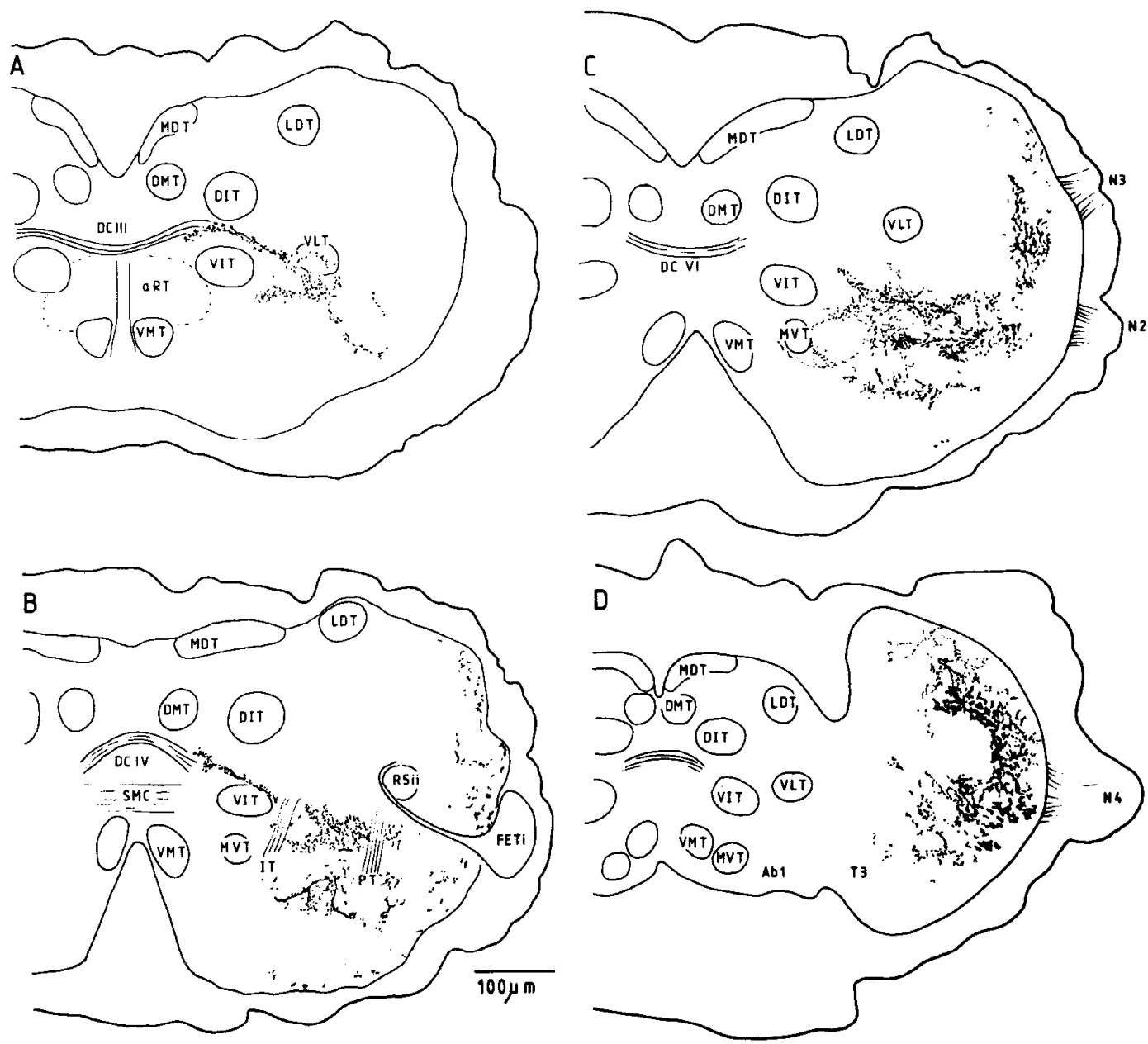

Figure 3. Transverse sections, at the levels indicated in Figure $2 A$, through a metathoracic ganglion in which the afferents from the femoral chordotonal organ were stained with cobalt. $A$, Anterior medial branches pass between VIT and DIT to DCIII. B, Posterior medial branches approach $D C I V$. Other branches are at the level of the I and $\mathrm{P}$ tracts, with a few branches at the lateral boundary of the neuropile, dorsal to the primary neurite of FETi. $C$, Extensive branches occur at the lateral boundary of the neuropile and at the level of VIT and $M V T$. D. Most dorsal branches are in the lateral neuropile. Each drawing is the composite of 4 adjacent $10 \mu \mathrm{m}$ sections. Abbreviations: $A b 1$, abdominal ganglion 1 ; $a R T$, anterior ring tract; $D C I I I, I V, V I$, dorsal commissures III, IV, and VI; DIT, dorsal intermediate tract; $D M T$, dorsal median tract; FETi, fast extensor tibiae motor neuron; $I T$, I tract; $L D T$, lateral dorsal tract; $M D T$, median dorsal tract; $M V T$, median ventral tract; $P T$, perpendicular tract; $S M C$, superior median commissure; R5ii, root of nerve $5 ; T 3$, neuromere of third thoracic segment; VIT, ventral intermediate tract; VLT, ventral lateral tract; $V M T$, ventral median tract.

cxtension by increascd flexor activity. The question to be answered, therefore, is whether these effects in the motor neurons and interneurons are mediated by direct connections of the FCO afferents.

\section{Connections with motor neurons}

To establish, using physiological criteria, whether afferents from the FCO connect directly with leg motor neurons, the following experimental procedure was adopted. A microelectrode was placed in the cell body of an afferent at the FCO and a second in the cell body of a flexor tibiae motor neuron in the metathoracic ganglion. A pair of extracellular electrodes on nerve 5 near the ganglion monitored the spikes in the afferent so that its conduction velocity could be determined.

A depolarizing pulse of current injected into an impaled afferent initiates spikes that travel toward the metathoracic ganglion, where they evoke depolarizing synaptic potentials in the impaled flexor tibiae motor neuron (Fig. 7). When the spikes are at frequencies below about $50 \mathrm{~Hz}$, it is clear that a depo- larizing potential follows cach spikc, cven though thcy are superimposed on a fluctuating background of other synaptic potentials (Fig. 7, $A, B$ ). When the frequency of spikes rises to between 60 and $110 \mathrm{~Hz}$, the depolarizing potentials that follow each spike begin to sum and hold the membrane depolarized for as long as the spikes persist (Fig. 7,C,D). With higher currents the frequency of afferent spikes and the evoked depolarization show an initial peak response, followed by a plateau (Fig. $7 E$ ). Finally, with still higher currents, which evoke spikes at approximately $300 \mathrm{~Hz}$, the spike-generating mechanism of the afferent soon fails and, as a consequence, the membrane potential of the motor neuron rapidly repolarizes (Fig. $7 F$ ).

In single sweeps on expanded time scales, each afferent spike is clearly seen to be followed by a depolarizing potential (Fig. $8 A$ ) that can be as large as $2.5 \mathrm{mV}$, depending on the afferent and the motor neuron being recorded. The synaptic potentials occur at a constant latency, as is shown by their superimposition in sweeps of the oscilloscope triggered by the afferent spikes (Fig. $8 B$ ). Estimates can now be made of the latency to the 

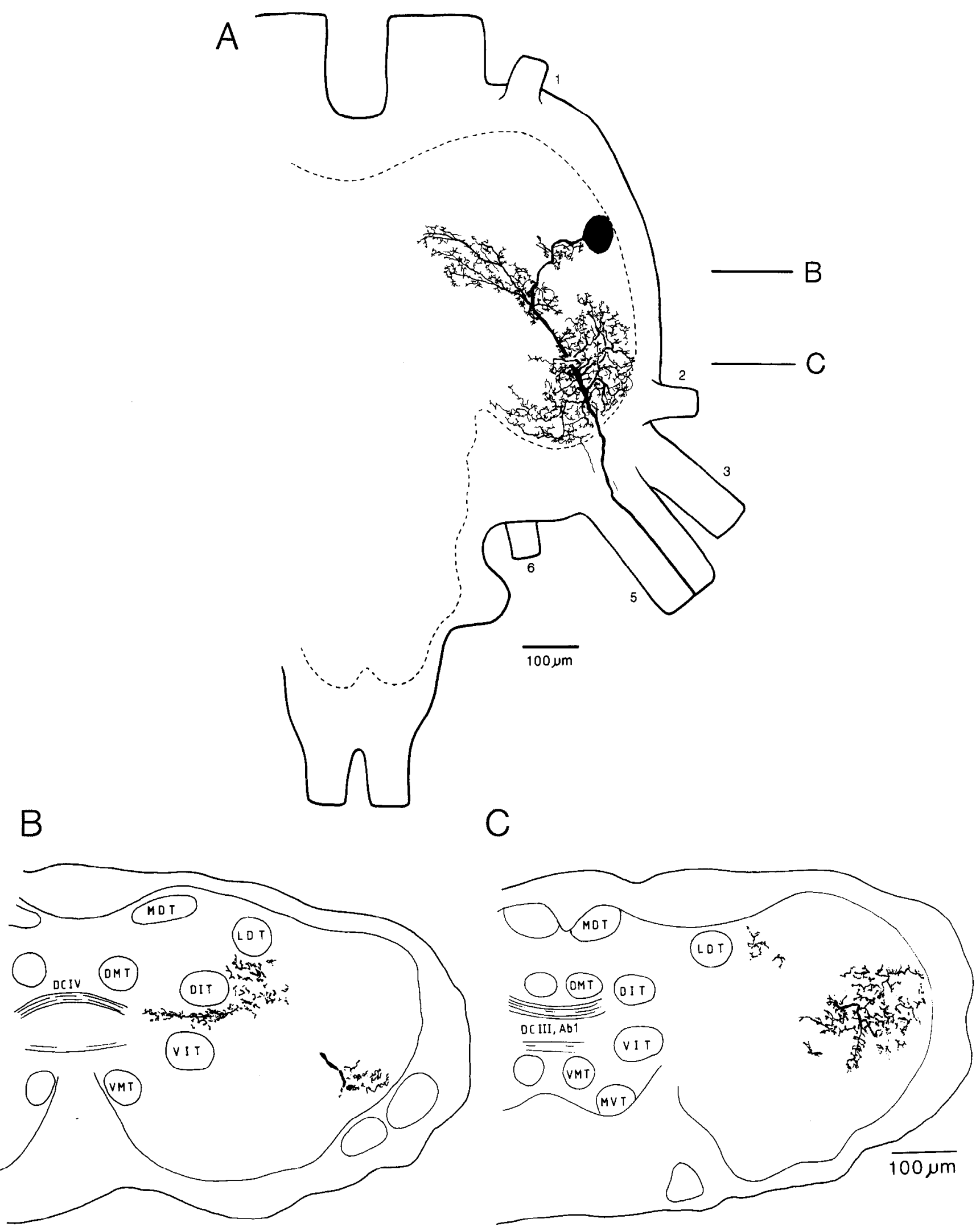

Figure 4. Morphology of a flexor tibiae motor neuron that is excited by a movement of the FCO apodeme equivalent to an extension of the femorotibial joint. $A$. Drawing of the right half of a whole-mount of the metathoracic ganglion viewed dorsally and with anterior at the top. A medial series of branches and a more posterior and lateral series are prominent. $B, C$, Transverse sections at the planes indicated in $A$. $B$, Section through the medial branches when they pass dorsal to VIT and approach $D C I V$. $C$, More posterior branches in the lateral neuropile at the level of $D I T$ and $V I T$, with a few branches lateral to $L D T$. Each drawing is the composite of 4 adjacent $10 \mu \mathrm{m}$ sections. 

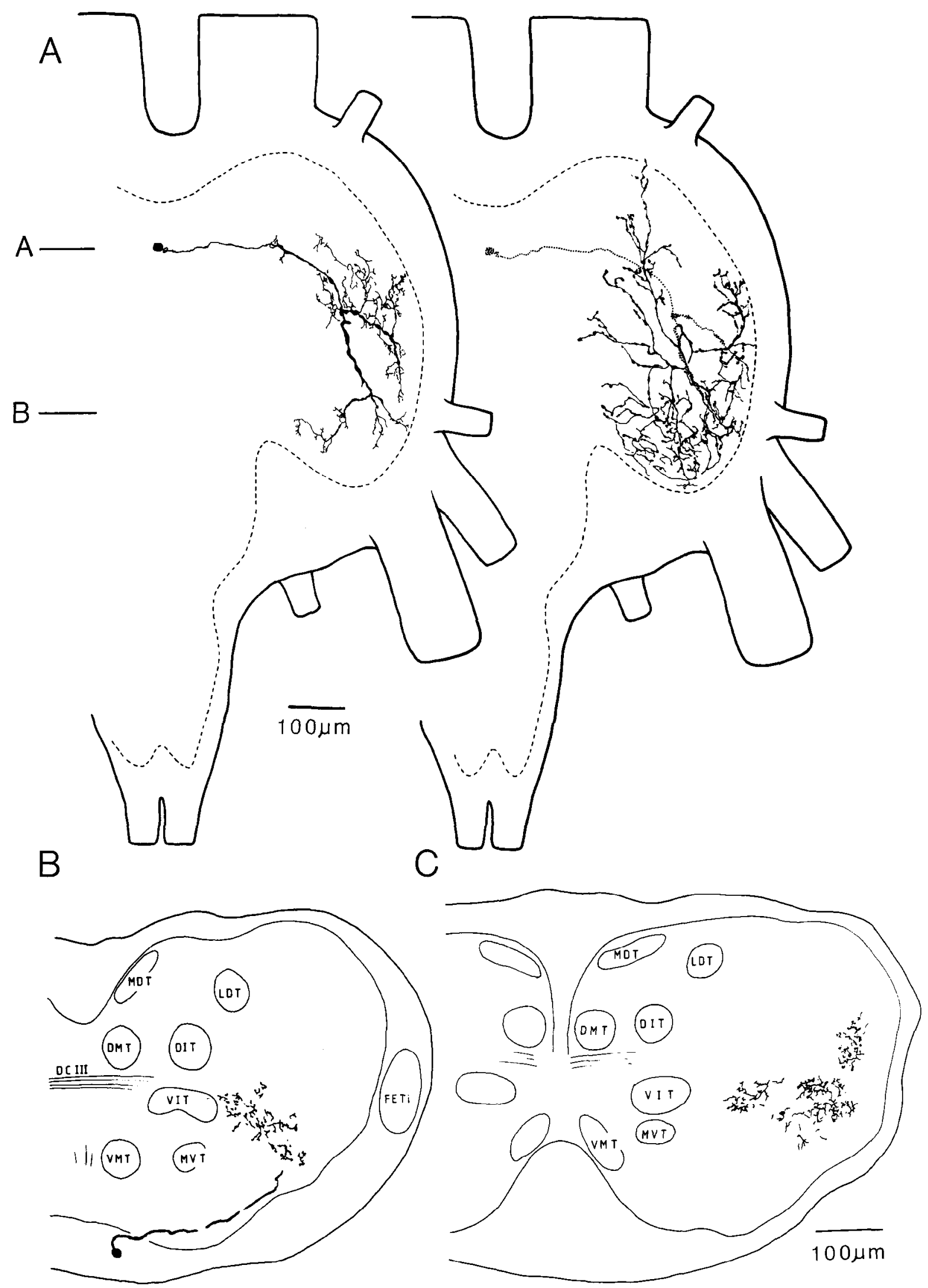

Figure 5. Morphology of a spiking local interneuron in the midline group that is excited by a movement of the FCO apodeme equivalent to an extension of the femorotibial joint. $A$, Branches of the interneuron are drawn in 2 parts: on the left, the sparse ventral branches, and on the right, the more extensive and varicose dorsal branches linked to the (stippled) ventral branches by a single process. $B, C$, Transverse sections at the planes indicated in $A . B$, Cell body, primary neurite, and branches close to VIT. $C$, More posterior branches in the lateral neuropile at the level of VIT Each drawing is the composite of 4 adjacent $10 \mu \mathrm{m}$ sections. 

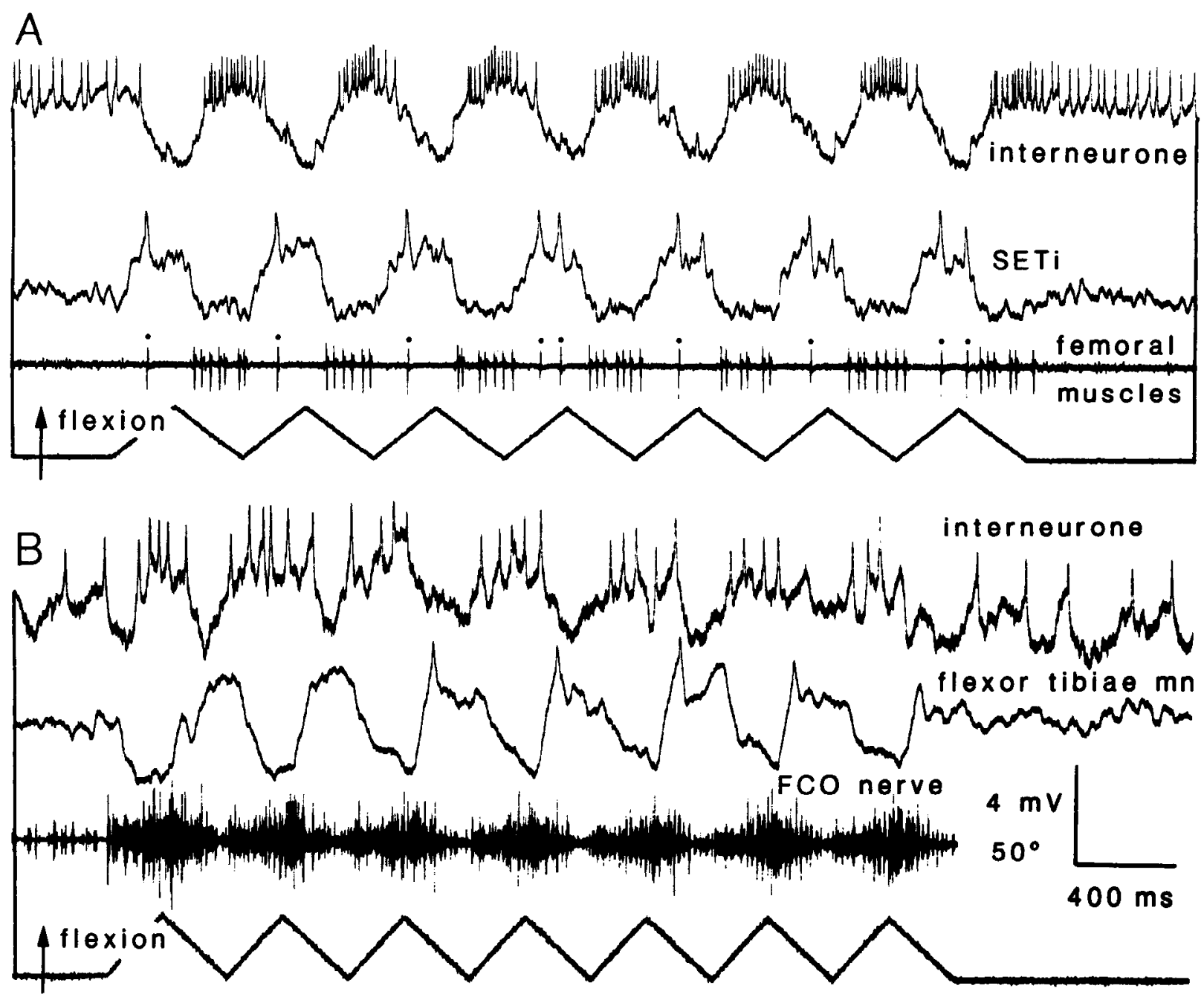

Figure 6. Effects of moving the FCO apodeme on spiking local interneurons and motor neurons in the metathoracic ganglion. $A$, Movements of the apodeme at $4 \mathrm{~Hz}$ that correspond to flexions (upwards on fourth trace) and extensions (downwards) of the tibia. A flexion inhibits 1 spiking local interneuron (first trace) and, at the same time, excites the slow extensor tibiae motor neuron (second trace). The spikes of this motor neuron are also monitored in the femur (dots), where they alternate with spikes from a flexor tibiae motor neuron. An extension movement excites the interneuron and inhibits the motor neuron. B, Second spiking local interneuron (first trace) and a flexor tibiae motor neuron (second trace). A flexion movement excites this interneuron but inhibits the flexor motor neuron, with extension having the opposite effects. An extracellular recording from the FCO nerve shows responses to both directions of movement. The movement of the apodeme in this and subsequent figures is calibrated in the equivalent angular movement of the femorotibial joint $\left(500 \mu \mathrm{m}\right.$ displacement $\left.=40^{\circ}\right)$. The starting point for all the movements corresponds to a femorotibial angle of $80^{\circ}$.

synaptic potential once the afferent spike has entered the ganglion. In the example shown in Figure 8, the afferent spike is conducted at a velocity of $3.4 \mathrm{msec}^{-1}$, as measured over a distance of $21 \mathrm{~mm}$ between the intracellular electrode in the afferent cell body and the extracellular electrodes on nerve 5 . From the time the spike is recorded in nerve 5 to the appearance of the synaptic potential, $2.3 \mathrm{msec}$ elapses. The electrodes on nerve 5 are $3 \mathrm{~mm}$ from the ganglion, so that $0.9 \mathrm{msec}$ of this time is used for conduction of the spike, leaving a central delay of 1.4 msec. This delay consists of the time it takes for the spike, once it has entered the ganglion, to reach the synaptic sites on the afferent terminals, for synaptic transmission, and for the synaptic potential to reach the electrode in the soma. The delay to a synaptic potential in a flexor tibiae motor neuron, measured in this way, has an average value of $1.5 \mathrm{msec}$ (range, 1.2-1.9 msec; $n=8$ ). Afferent neurons with the slowest conduction velocities $\left(2.8 \mathrm{msec}^{-1}\right)$ evoke synaptic potentials with the longest central latency, while those with the fastest velocities $\left(3.7 \mathrm{msec}^{-1}\right)$ evoke potentials with the shortest latency, which emphasizes the contribution of the conduction time within the ganglion to the measured central delay.

At the resting potential of the motor neuron shown in Figure 9, each afferent spike evokes a depolarizing potential of approximately $1 \mathrm{mV}$ in amplitude (Fig. 9,A,D) and the summed depolarization elicited by spikes at $60 \mathrm{~Hz}$ is about $2.5 \mathrm{mV}$. If the motor neuron is hyperpolarized with a steady current injected through the recording microelectrode, the amplitude of the depolarization produced by spikes at $60 \mathrm{~Hz}$ is now almost $5 \mathrm{mV}$ (Fig. 9B). Individual potentials can almost double in size, depending on the hyperpolarizing current applied (Fig. 9D). If the motor neuron is now depolarized instead, the amplitude of the evoked potentials is decreased (Fig. 9,C,D), but afferent spikes are now able to elicit motor spikes (Fig. 9 C).

Taken together, this evidence indicates that some afferents from the FCO synapse directly upon flexor tibiae motor neurons and evoke chemically mediated EPSPs. 

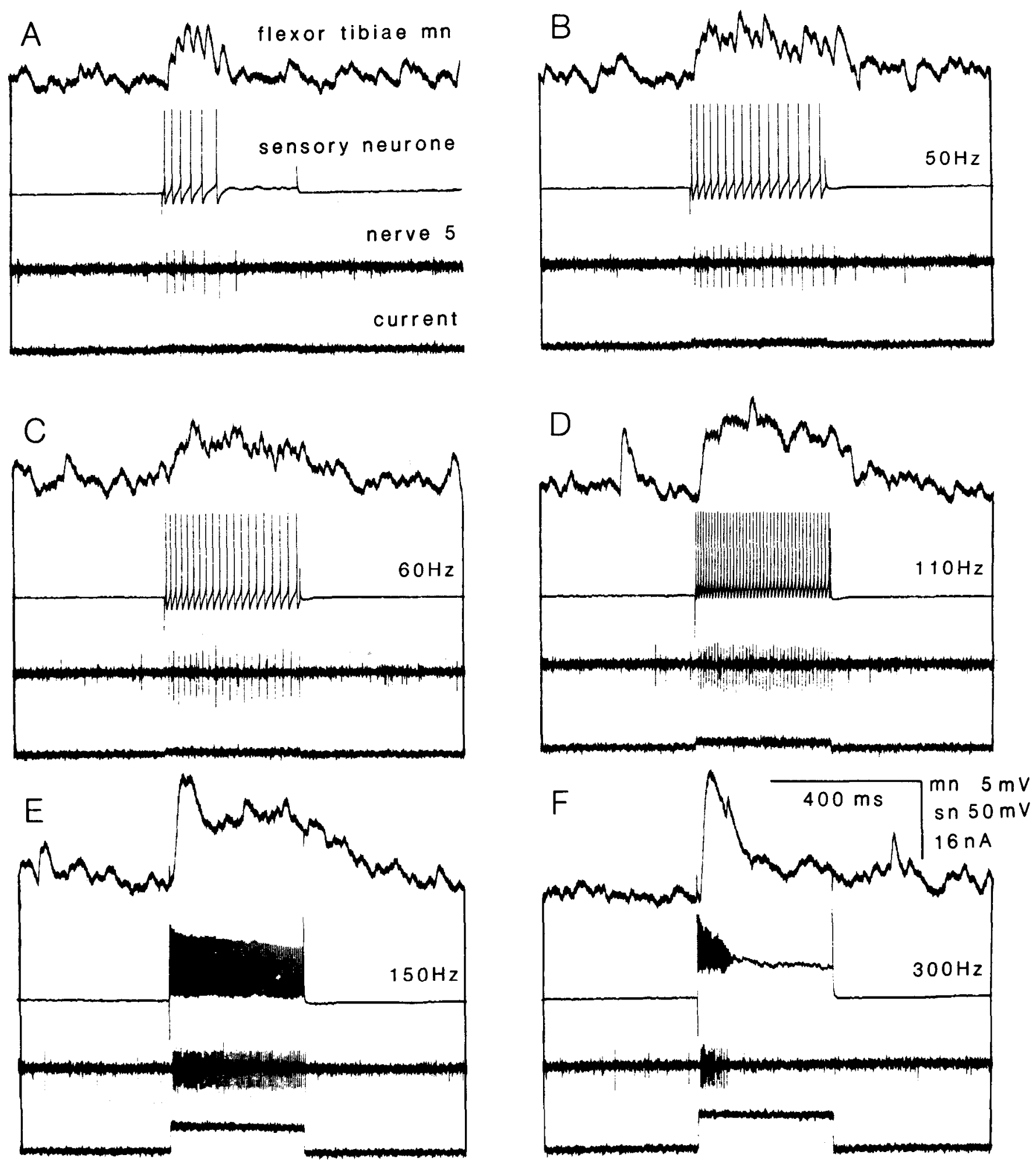

Figure 7. Connections between a sensory neuron in the chordotonal organ and a flexor tibiae motor neuron. Intracellular recordings are from the cell body of the motor neuron and from the cell body of the afferent in the chordotonal organ. The afferent spike is recorded in nerve 5,21 mm from the FCO and $3 \mathrm{~mm}$ before it enters the metathoracic ganglion. $A$, Depolarizing current is injected into the sensory neuron to evoke 6 spikes, each of which is followed by an extracellular spike in the nerve and an EPSP in the motor neuron. $B-E$, Increasing current injected into the scnsory neuron evokes spikes of progressively higher frequencies and larger depolarizations of the motor neuron. $F$, With high currents, the spike mechanism of the sensory neuron fails and is accompanied by a failure of the evoked depolarization in the motor neuron.

An afferent may connect with more than 1 motor neuron. For example, 2 flexor tibiae motor neurons, recorded simultaneously, show parallel changes in their membrane potentials, with many synaptic potentials in common, when the $\mathrm{FCO}$ apodeme is moved (Fig. 10A). Low-amplitude vibrations of the $\mathrm{FCO}$ evoke an almost perfectly matching scquence of EPSPs in the 2 motor neurons (Fig. 10B). Simultaneous intracellular recordings show that an afferent spike is followed by an EPSP in 2 


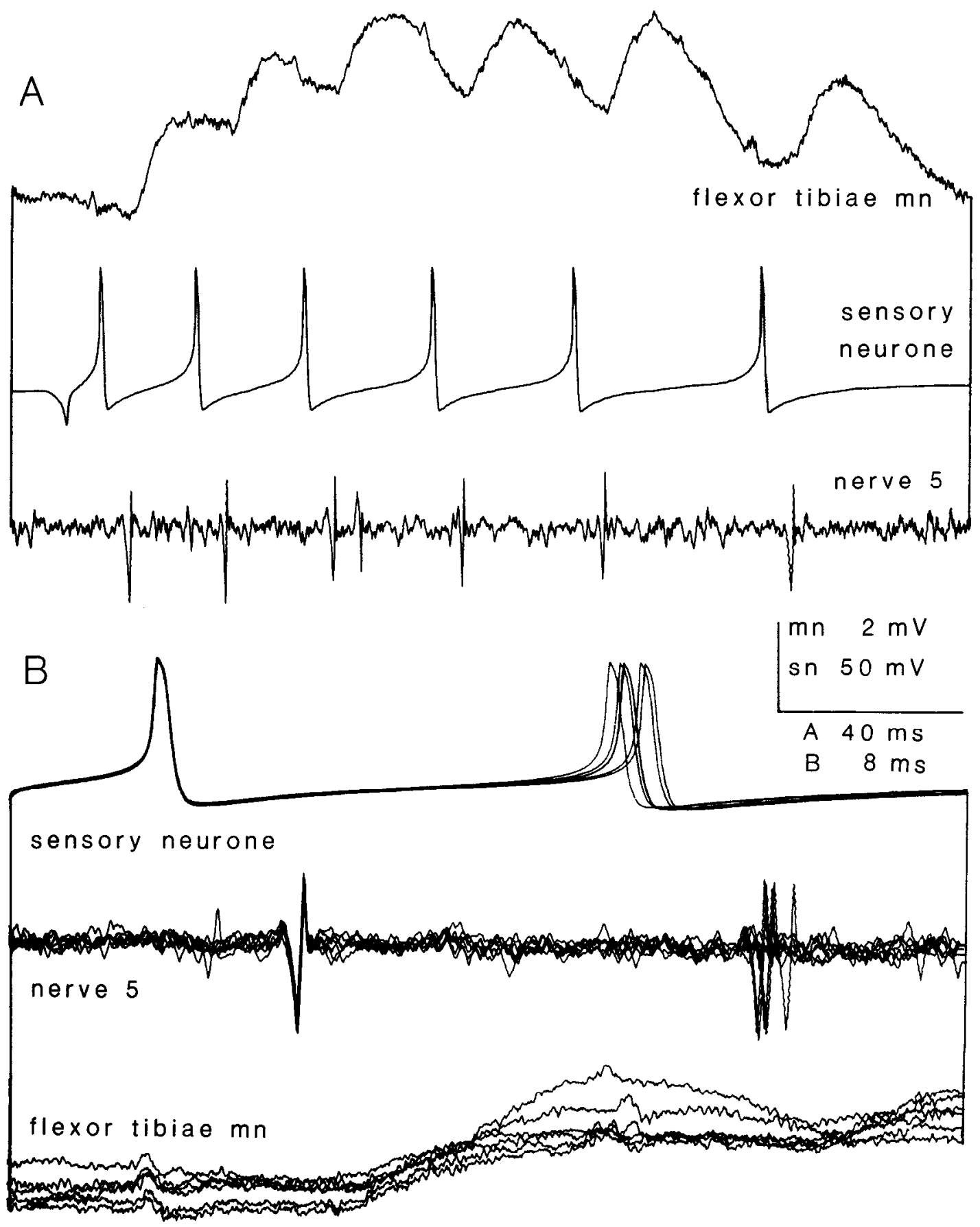

Figure 8. Latency of the synaptic potential in a flexor tibiae motor neuron. The recording arrangement is as in Figure 7. A, Each afferent spike (second trace) cvoked by a $400 \mathrm{msec}$ pulsc of current (bcginning at the arrow) injected into the sensory neuron is followed by a depolarizing synaptic potential recorded intracellularly in the motor neuron. The third trace monitors the spike of the sensory afferent in nerve 5 . $B$, Six superimposed sweeps of an oscilloscope triggered by the sensory spike recorded intracellularly show that the potentials occur with a constant latency.

flexor motor neurons with the same central latency of $1.5 \mathrm{msec}$ (Fig. 10C). A second afferent in the same locust also evokes EPSPs with differing amplitudes but the same latency in both neurons (Fig. 10D). Using this arrangement of electrodes, 1 afferent was shown to evoke EPSPs with the same latency in 3 flexor motor neurons in the same locust. First, 2 fexors were impaled simultaneously, and then 1 of the electrodes was moved to a third motor neuron. These $\mathbf{3}$ flexor motor neurons are not electrically or synaptically coupled to each other. These experiments do not, however, address the issue of whether connec- tions made by the afferents vary for the different types of flexor motor neurons.

An afferent that evokes an EPSP in a flexor has no effect on the antagonistic slow extensor tibiae motor neuron recorded at the same time. Different afferents do, however, evoke EPSPs in the slow extensor motor neuron, with central latencies of 1.5 msec. They, in turn, have no effect on the flexor tibiae motor neurons that have been sampled. It can therefore be suggested that one population of afferents excites flexor motor neurons and a different population excites the extensor motor neuron. 

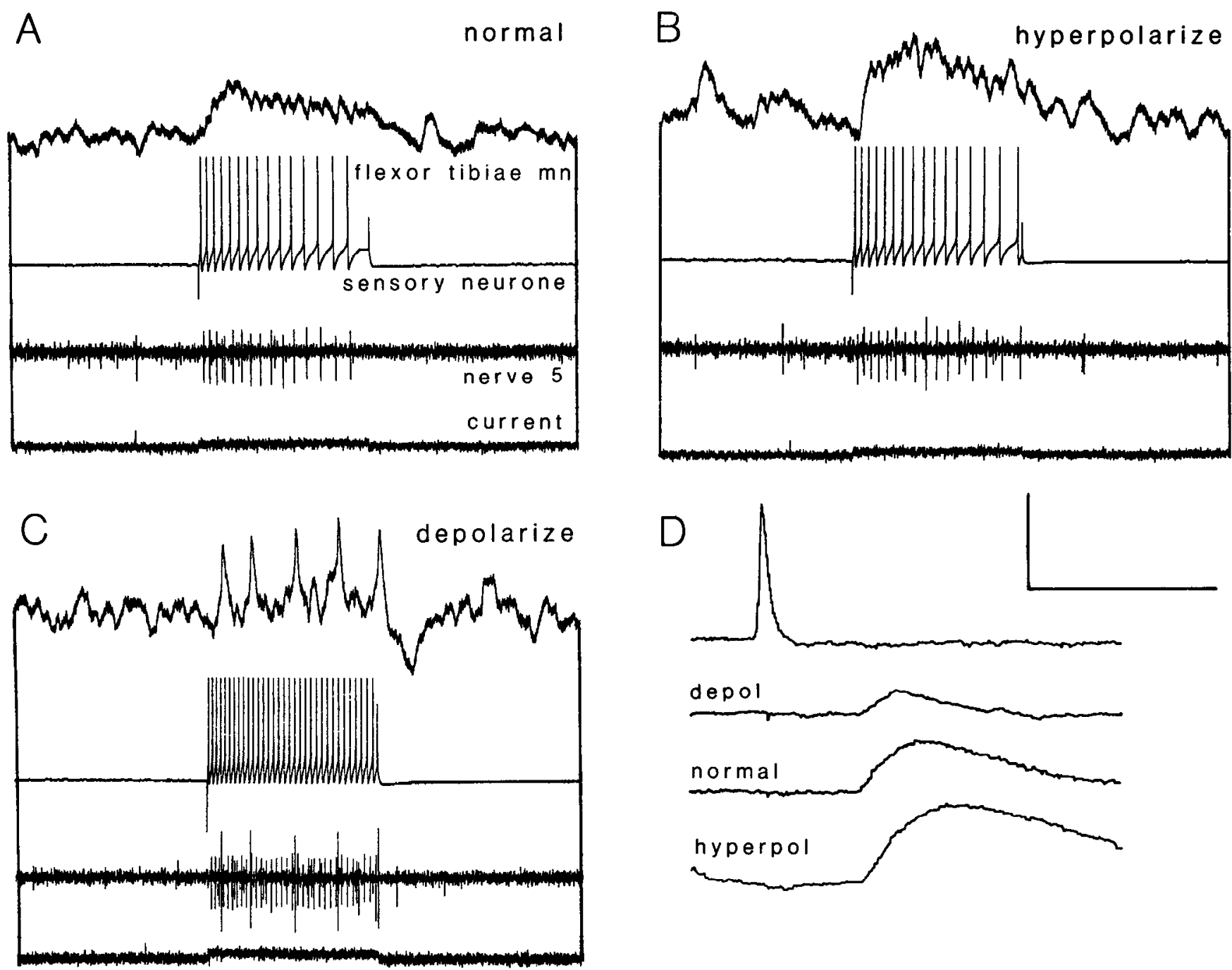

Figure 9. Changing the membrane potential of the flexor motor neuron alters the amplitude of a synaptic potential from a chordotonal afferent. $A$, Motor neuron is at its resting potential and afferent spikes evoke potentials that sum to produce a depolarization of $2.5 \mathrm{mV} . B$, Hyperpolarizing current is injected into the motor neuron, and the afferent spikes now evoke a depolarization of $5 \mathrm{mV}$. $C$, Motor neuron is depolarized and afferent spikes are now able to evoke spikes in the motor neuron that are also seen in the recording from nerve 5 . D. Averaged synaptic potentials at different membrane potentials of the motor neuron in another locust. Each trace is the average of 64 occurrences of the EPSP triggered by the afferent spike. Calibration: $A-C$, motor neuron $5 \mathrm{mV}$, sensory neuron $50 \mathrm{mV}$, current $16 \mathrm{nA} ; D$, motor neuron $2 \mathrm{mV}$, sensory neuron $50 \mathrm{mV}$; horizontal $A-C, 400 \mathrm{msec} ; D, 22.5 \mathrm{msec}$.

\section{Connections with spiking local interneurons}

A simultaneous recording of a spiking local interneuron and a flexor tibiae motor neuron shows that both undergo parallel changes in their membrane potentials when the FCO apodeme is moved (Fig. 11A). Both neurons are hyperpolarized by a movement corresponding to flexion of the femorotibial joint, and, during an extention, both are depolarized sufficiently by common inputs to produce spikes. When the manipulator holding the receptor apodeme is gently tapped, both the pattern and number of EPSPs are almost the same in the 2 neurons (Fig. $11 B$ ). However, only inputs from the chordotonal organ evoke this pattern of common synaptic potentials. When hairs on the ventral surface of the tibia are touched, the interneuron is depolarized and spikes, but the motor neuron is unaffected (Fig. $11 C$ ). The 2 neurons are not linked to each other by direct electrical or chemical synapses, as passing current directly into one to evoke spikes is without effect on the other. The coupling between them is thus entirely at the presynaptic level, resulting from spikes generated by afferents from the chordotonal organ.

On an expanded time scale, common EPSPs in an interneuron and a motor neuron are clearly seen to be associated with bursts of spikes from the FCO afferents (Fig. 12A). Setting a window circuit to select 1 amplitude of these extracellularly recorded spikes and using this signal to trigger a signal averager shows that an EPSP occurs with the same latency in both neurons (Fig. 

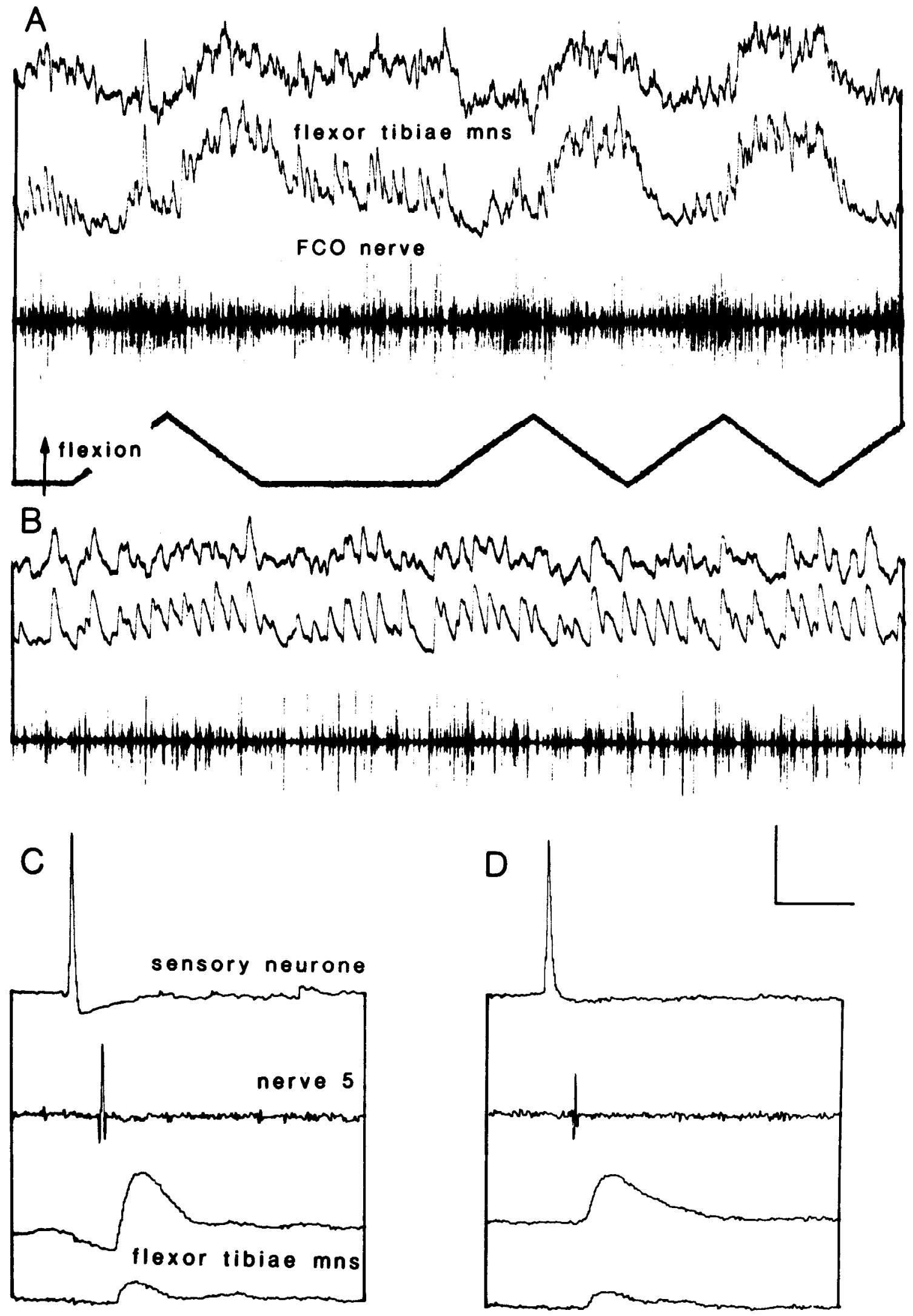

in common. $B$, Apodeme of the FCO is held still, but so sensitive are the receptors that they respond to the sound produced by the recording apparatus (spikes of FCO nerve on third trace). Most of the EPSPs are common to both neurons. C,D, Intracellular recordings from FCO afferents (first trace) and from 2 flexor tibiae motor neurons (third and fourth traces) in 2 locusts. The afferent spikes are also recorded in nerve 523 mm from the FCO and $4 \mathrm{~mm}$ from the metathoracic ganglion. Spikes of 2 afferents $(C$ and $D)$ trigger a signal-averager. The displays are the result of 64 occurrences of a spike. Both motor neurons receive an EPSP with the same latency. Calibration: Vertical $A, B, 4 \mathrm{mV}, \mathrm{movement} 50^{\circ}$; $C, D$, sensory neuron $30 \mathrm{mV}$, motor neuron $2 \mathrm{mV}$; horizontal $A$, $400 \mathrm{msec} ; B, 200 \mathrm{msec} ; C, D, 22.4 \mathrm{msec}$. 

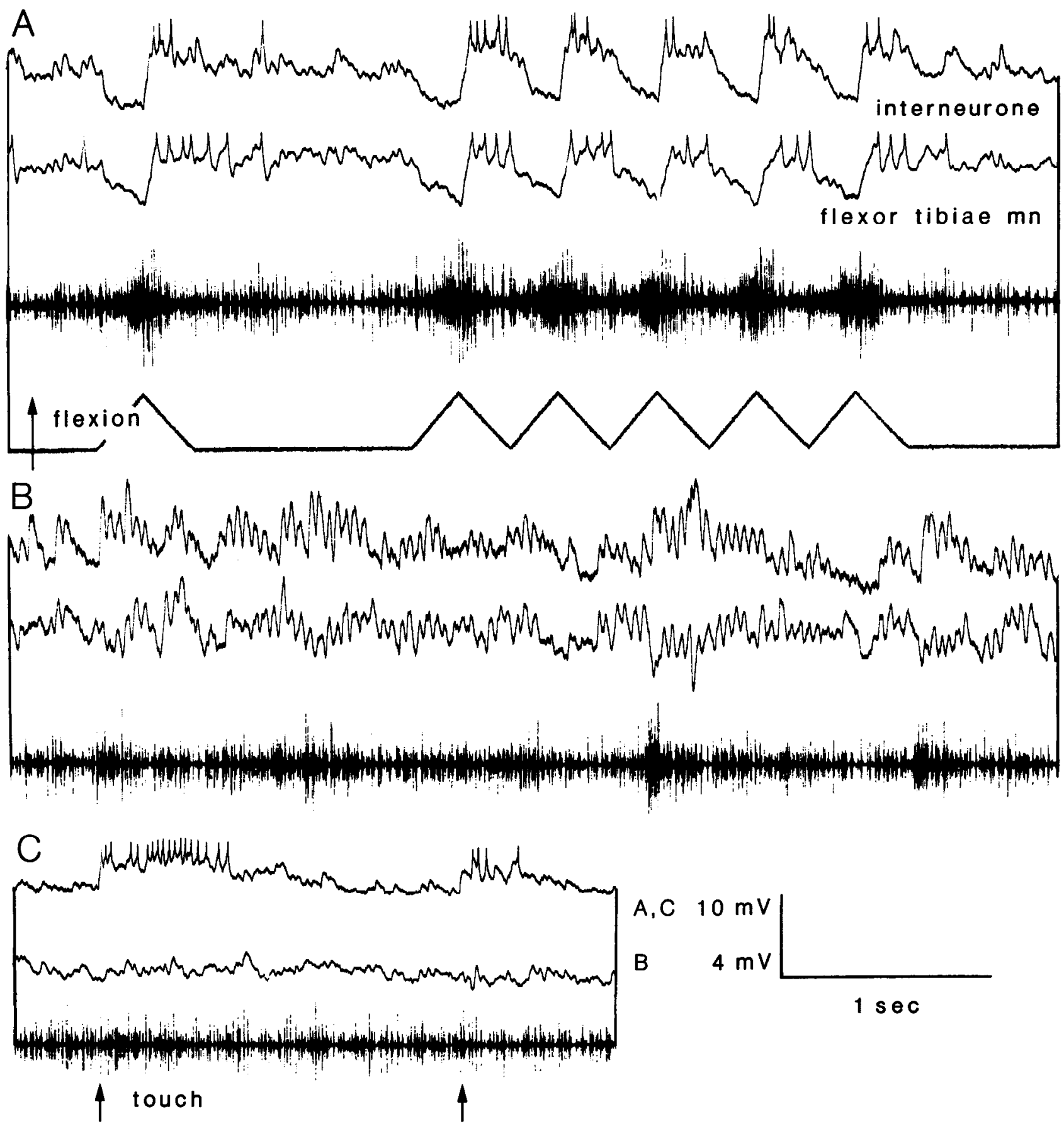

Figure 11. Parallel responses of a spiking local interneuron (first trace) and a flexor tibiae motor neuron (second trace) to movements of the chordotonal organ. A, Movement of the apodeme, which corresponds to a flexion of the femorotibial joint, inhibits both neurons, whereas a movement corresponding to extension excites them both. The majority of EPSPs are the same in both neurons, but the number of spikes they produce differs. $B$, Manipulator holding the FCO apodeme is gently tapped. The resulting vibration of the FCO evokes common EPSPs in both neurons. $C$, Touching hairs on the ventral surface of the tibia evokes a burst of spikes in the interneuron, but has no effect on the motor neuron.

$12 B)$. The EPSP in this particular interneuron has a faster rise time and larger amplitude than that in the motor neuron and sometimes leads to a spike, but the reverse can obtain (Fig. 12C).

Stimulating the FCO nerve electrically before it joins N5bl evokes EPSPs with the same latency in a spiking local interneuron and a flexor motor neuron (Fig. 12D). Altering the strength of the stimulus, which presumably has the effect of changing the number of afferents that are excited, changes the amplitude of the compound EPSPs (Fig. 12E). This confirms the previous observation that several afferents synapse on a flexor motor neuron, and additionally shows that several also synapse on an interneuron. The compound EPSPs in either neuron can evoke spikes. At certain stimulus intensities, the EPSP in the motor neuron is curtailed by a hyperpolarizing IPSP (Fig. 12E). This potential always has a longer latency, by some $1-2$ msec, than the EPSP, and is not accompanied by a hyperpolarization of the interneuron. 

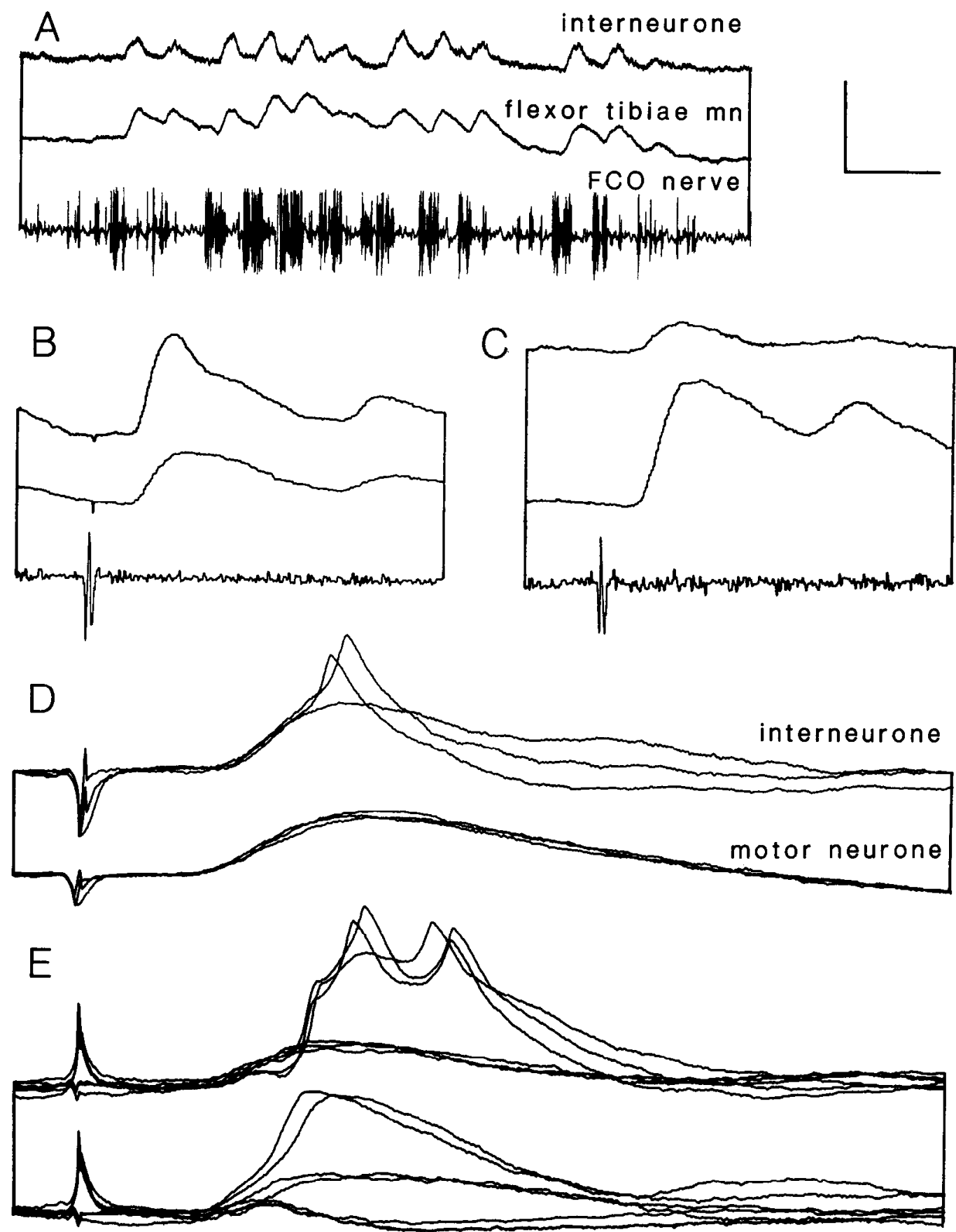

Figure 12. Afferents from the FCO synapse on a spiking local interneuron and a flexor tibiae motor neuron. $A$, Common EPSPs in a spiking local interneuron (first trace) and a flexor tibiae motor neuron (second trace) recorded simultaneously. Each EPSP is preceded by a burst of spikes in the FCO nerve (third trace). $B, C$, A window circuit is used to select spikes of a particular amplitude from an extracellular recording of the FCO nerve (third trace) and to trigger a signal-averager. $B$, An afferent spike evokes a large EPSP in the interneuron and a smaller one in the motor neuron with the same latency. $C$, In another locust, a sensory spike evokes a large EPSP in the motor neuron and a smaller one in the interneuron, again with the same latency. $D, E$, Electrodes on the FCO nerve are used for electrical stimulation and the current is varied. $D$, Stimulus evokes an EPSP with the same latency in both neurons. EPSPs in the interneuron twice evoke a spike. $E$, Increasing the stimulus strength leads to a spike in both neurons. With a further increase, the stimulus now evokes an IPSP in the motor neuron, with a longer latency than the EPSPs. Each record is from a different locust in which an interneuron and a motor neuron were impaled simultaneously. Calibration: Vertical $A, 2 \mathrm{mV} ; B, C, 2 \mathrm{mV} ; D, E$, $4 \mathrm{mV}$; horizontal $A, 200 \mathrm{msec} ; B, C, 22.4 \mathrm{msec} ; D, E, 7.8 \mathrm{msec}$.

\section{Discussion}

\section{Connections of the afferents}

Some afferents from the femoral chordotonal organ make excitatory connections with both motor neurons and spiking local interneurons. Are these connections monosynaptic? In ex- periments in which an afferent cell body is impaled, there is a clear causal relationship between afferent spikes induced by the injection of current and EPSPs in a motor neuron. Each afferent spike is followed by an EPSP at a consistent and short latency, even at frequencies as high as $100 \mathrm{~Hz}$. The dependence of the EPSP amplitude on the membrane potential of the postsynaptic 
neuron indicates that the synapse operates chemically. The central latency to an EPSP is about $1.5 \mathrm{msec}$, of which $0.1-0.2 \mathrm{msec}$ is due to the conduction of the spike to the afferent terminals, and a similar amount to the conduction of the synaptic potential to the recording electrode in the soma. The remaining time would seem to be sufficient for only 1 chemical synapse in the pathway. Simultaneous recordings from interneurons and motor neurons show that synaptic potentials follow an afferent spike with the same consistent latency in both neurons. Therefore, whatever conclusions are drawn about the connection with a motor neuron must apply equally to the connection with an interneuron. The central delay is similar to that observed at the chemical synapses between other afferents and central neurons in this ganglion; for example, the wing stretch receptor (Burrows, 1975), hair afferents (Siegler and Burrows, 1983), and afferents from the movable spurs on the tibia (Burrows and Pflüger, 1986). It is somewhat longer than the delay measured between 2 neurons within the central nervous system, where the recording electrodes may be closer to the synaptic sites (Burrows and Siegler, 1982; Robertson and Pearson, 1985; Selverston et al., 1985).

The anatomy of the afferents, motor neurons, and spiking local interneurons is consistent with direct connections, because their projections overlap. Indeed, there are striking similarities in some of the projections, most notably the medially directed branches that pass between VIT and DIT and run toward DCIV. The combined physiological and anatomical evidence strongly indicates that the connection to the motor neurons and interneurons is direct, but final confirmation awaits the demonstration of synaptic contacts with the electron microscope.

Not all afferents excited by an extension movement synapse upon 1 particular flexor motor neuron; this emphasizes the different features of the stimulus that are encoded across the population of afferents, and the different functional properties of individual motor neurons. Each afferent can nevertheless synapse upon as many as 3 flexor motor neurons from the excitatory pool of some 9 (Phillips, 1981) neurons. The profusion of common synaptic potentials found in some motor neurons during movements of a leg would be explained if these afferents made more widespread connections (Burrows and Horridge, 1974). Only a small proportion of the spiking local interneurons in the midline group receive inputs from FCO afferents and these code extension, flexion, or a movement in both directions. Thus, a particular flexor motor neuron or a spiking local interneuron receives inputs from several afferents, and each afferent, in turn, makes connections with several motor neurons and interneurons. What must now be determined is the pattern of connectivity of the different types of afferents with the different types of motor neurons within one pool. For example, do afferents that code position connect with tonic motor neurons, and those that code movement connect with phasic motor neurons?

Motor neurons and some interneurons are inhibited, as well as excited, by movements of the FCO apodemc. Nonc of the inhibitory effects are, however, mediated by direct afferent connections. Searching for inhibitory potentials linked to an afferent spike by a variety of means, including signal-averaging, has always failed. The involvement of more complex pathways is also suggested by the longer latency to an inhibitory potential evoked by electrical stimulation of the afferent axons. The reason inhibitory potentials can be linked to spikes evoked by electrical stimulation and not to those evoked by movement is presumably that many afferents will be discharged synchronously when the nerve is stimulated electrically.

\section{Reflex pathways}

The effects of the FCO afferents on both motor neurons and interneurons are powerful. Movements of the apodeme that correspond to small angular changes of the femorotibial joint can evoke bursts of spikes in both classes of neurons. An extension movement, for example, excites many flexor motor neurons, inhibits the antagonistic extensors, and, in parallel, excites some interneurons and inhibits others. The effect on the motor neurons is to evoke a classical resistance reflex that would oppose the imposed movement. Why, then, should there be parallel connections with interneurons? Their possible role could be to mediate the inhibitory arm of the resistance reflex by inhibiting the antagonistic motor neurons - for example, those interneurons excited by an imposed extension of the tibia could inhibit the extensor tibiae motor neurons. This idea is attractive for 3 reasons. First, the pattern of spikes in some of these spiking local interneurons matches the patterns of inhibition in the antagonistic motor neurons (see Fig. 2). Second, spiking local interneurons in the midline group make inhibitory connections with motor neurons, among them the slow extensor tibiae motor neuron (Burrows and Siegler, 1982, 1984). It is, however, puzzling that few connections between these interneurons and motor neurons have been found. Third, some of these interneurons stain with antibodies raised against GABA, and their inhibitory actions are blocked by picrotoxin (Watson and Burrows, 1987).

It seems likely, however, that the spiking local interneurons exert a wider influence than this. They could provide the point at which the afferent signals, once integrated to code a particular feature of joint movement or to force changes, are distributed more widely to other interneurons. Likely targets are nonspiking local interneurons and long interneurons. The nonspiking interneurons would then, by virtue of their connections with sets of motor neurons (Burrows, 1980), call forth a coordinated response of several muscles that underlie interjoint reflexes. The long interneurons would distribute the information to ganglia controlling other legs, so that an appropriate adjustment of the gait is made in response to any disturbance or change in load detected by one leg.

These local reflex pathways must, however, permit voluntary movements in the presence of any direct afferent excitation of the motor neurons that would oppose such a movement. In stick insects, the system controlling the position of the femorotibial joint, of which the $\mathrm{FCO}$ is an essential part, is active during walking (Cruse and Pflüger, 1981). A voluntary extension of the tibia would thus be expected to excite certain FCO afferents, which would then excite flexor tibiae motor neurons. Vertebrate la afferents receive presynaptic inhibition from afferent pathways activated when other joints move (Eccles, 1964; Rudomin, 1980). In crabs, the central terminals of nonspiking afferents from the coxal receptors are depolarized in time with the centrally generated pattern of motor output corresponding to walking - a mechanism that could ensure that the afferent signals are heeded only at the appropriate phase of the movement (Sillar and Skorupski, 1986). It is not known whether the FCO afferents can be inhibited presynaptically, although other types of locust afferents do receive input synapses on their terminals (Altman et al., 1980; Watson and Pflüger, 1984). A fur- 
ther role for the spiking local interneurons could therefore be to mediate presynaptic inhibition of particular afferents in a way that is similar to the inhibition caused by local and long interneurons in the crayfish (Kirk and Wine, 1984).

\section{Spiking local neurons participate in 2 classes of reflex}

Spiking local interneurons in the same midline population also participate in postural reflexes of the leg elicited by exteroreceptors (Burrows and Siegler, 1982; Siegler and Burrows, 1986). They do this by virtue of the direct inputs they receive from exteroreceptors and through the direct output connections they make with motor neurons. Are there features common to the actions of these interneurons in tactile reflexes and in proprioceptive reflexes? In tactile reflexes, the afferents from individual hairs do not synapse directly upon the motor neurons, so that all motor effects are mediated by interneurons. The shortest pathway results in inhibition of motor neurons (sensory neuronspiking local interneuron-motor neuron), whereas excitation of motor neurons results from more complex pathways, in which other local interneurons are implicated. By contrast, the shortest pathway in proprioccptive reflexes results in cxcitation of motor neurons without the participation of interneurons (sensory neuron-motor neuron), whereas inhibition results from more complex pathways, in which local interneurons are again implicated. The spiking local interneurons are therefore involved in both the inhibitory and excitatory loops of the tactile reflexes, but only in the inhibitory loop of the proprioceptive reflexes. Their roles in the 2 classes of reflex are not completely separable, however, because many receive inputs from both extero- and proprioceptors (Burrows, 1985; see also Fig. 11). The 2 classes of inputs can elicit the same reflex movements because of their convergence onto the same spiking local interneurons. For example, depression of the tarsus can be elicited by FCO afferents when the tibia is extended, and by hairs or spurs on the tibia.

\section{Do these pathways explain known features of proprioceptive control?}

The 2 competing demands of proprioceptive control of movement are that it be reliable yet modifiable. The direct afferent pathway to motor neurons explains the reliability of motor responses to imposed, high-frequency movements of a joint. The connections with interneurons suggest possible explanations for variability. For example, flexor motor neurons are excited by either an imposed extension or flexion movement during active seeking movements of the leg (Zill, 1985b). This could result from the activation of local interneurons that are themselves excited by both extension and flexion movements (Burrows, 1985). Although the present results suggest some means by which this type of variability, and the reversal of reflexes (Bässler, 1976), could occur, they do not detail the mechanism. Presumably this resides in the differing efficacy of the output of the interneurons that is determined in large part by the changing background of synaptic potentials in neurons with which they interact.

One result the connections described here cannot explain is the reported rapidity of the reflexes elicited by the locust FCO on hind leg motor neurons, and on muscles in other ipsilateral legs. Macmillan and Kien (1983) stimulated the FCO electrically in a standing locust, using pairs of implanted $50 \mu \mathrm{m}$ wires (note the size of the FCO in Fig. 1) and described potentials appearing in meso- and metathoracic flexor and extensor muscles only 5 msec later. Failure of these potentials to follow at stimulation frequencies of $30 \mathrm{~Hz}$ led them to suggest that the pathways were not monosynaptic. However, the minimum reflex time of the pathway measured here is almost $13 \mathrm{msec}$; spikes from the fastest FCO afferents take $7.8 \mathrm{mscc}$ to travcl the $25 \mathrm{~mm}$ to the metathoracic ganglion; there is a central delay of $1.5 \mathrm{msec}$; and the motor spikes take $3.5 \mathrm{msec}$ to reach the muscle and evoke a muscle potential (assuming a conduction velocity of $4 \mathrm{msec}^{-1}$ and a neuromuscular delay of $1 \mathrm{msec}$ ). The effects described by Macmillan and Kien can only be explained by assuming that the current they used to stimulate the FCO leaked and excited other neurons, possibly the motor neurons directly. The effects they ascribe to the FCO during walking are not, therefore, substantiated.

\section{References}

Altman, J. S., M. K. Shaw, and N. M. Tyrer (1980) Input synapses onto a sensory neurone revealed by cobalt-electron microscopy. Brain Res. 189: 245-250.

Bacon, J. P., and J. S. Altman (1977) A silver intensification method for cobalt-filled neurones in wholemount preparations. Brain Res. 138: 359-363.

Bässler, U. (1968) Zur Steuerung des Springens bei der Wanderheuschrecke Schistocerca gregaria. Kybernetik 4: 112.

Bässler, U. (1976) Reversal of a reflex to a single motoneuron in the stick insect Carausius morosus. Biol. Cybern. 24: 47-49.

Bässler, U. (1977) Sensory control of leg movement in the stick insect Carausius morosus. Biol. Cybern. 25: 61-72.

Bässler, U. (1983) Neural Basis of Elementary Behaviour in Stick Insects, Springer, Berlin.

Blight, A. R., and R. Llinás (1980) The non-impulsive stretch-receptor complex of a crab: A study of depolarization-release coupling at a tonic sensorimotor synapse. Phil. Trans. R. Soc. Lond. [Biol.] 290: 219-276.

Bräunig, P. (1985) Strand receptors associated with the femoral chordotonal organs of locust legs. J. Exp. Biol. 116: 331-341.

Brown, A. G. (1981) Organization in the Spinal Cord, Springer, Berlin.

Burns, M. D. (1974) Structure and physiology of the locust femoral chordotonal organ. J. Insect Physiol. 20: 1319-1339.

Burrows, M. (1975) Monosynaptic connexions between wing stretch receptors and flight motoneurones of the locust. J. Exp. Biol. 62: 189219.

Burrows, M. (1980) The control of sets of motoneurones by local interneurones in the locust. J. Physiol. (Lond.) 298: 213-233.

Burrows, M. (1985) The processing of mechanosensory information by spiking local interneurones in the locust. J. Neurophysiol. 54: 463478.

Burrows, M., and G. A. Horridge (1974) The organization of inputs to motoneurones of the locust metathoracic leg. Phil. Trans. R. Soc. Lond. [Biol.] 269: 49-94.

Burrows, M., and H. J. Pflüger (1986) Processing by local interneurons of mechanosensory signals involved in a leg reflex of the locust. $J$. Neurosci. 6: 2764-2777.

Burrows, M., and M. V. S. Siegler (1982) Spiking local interneurons mediate local reflexes. Science 217: 650-652.

Burrows, M., and M. V. S. Siegler (1984) The morphological diversity and receptive fields of spiking local interneurones in the locust metathoracic ganglion. J. Comp. Neurol. 224: 483-508.

Burrows, M., and M. V. S. Siegler (1985) The organization of receptive fields of spiking local interneurones in the locust with inputs from hair afferents. J. Neurophysiol. 53: 1147-1157.

Cruse, $H_{\text {., }}$ and H. J. Pflüger (1981) Is the position of the femur-tibia joint under feedback control in the walking stick insect? II. Electrophysical recordings. J. Exp. Biol. 92: 97-107.

Eccles, J. C. (1964) The Physiology of Synapses, Springer, Berlin.

Field, L. H., and M. Burrows (1982) Reflex effects of the femoral chordotonal organ upon leg motor neurones of the locust. J. Exp. Biol. 101: 265-285.

Field, L. H., and F. C. Rind (1981) A single insect chordotonal organ mediates inter- and intra-segmental leg reflexes. Comp. Biochem. Physiol. 68A: 99-102. 
Grosch, A., F. Callender, M. Petersen, C. Cokl, and K. Kalmring (1985) Vibration receptors of larvae and of imagines in locusts: Location on the legs, central projections and physiology. In Acoustic and Vibrational Communication in Insects, K. Kalmring and N. Elsner, eds., pp. 151-161, Paul Parey, Berlin.

Heitler, W. J., and M. Burrows (1977) The locust jump. II. Neural circuits of the motor programme. J. Exp. Biol. 66: 221-241.

Hofmann, T., U. T. Koch, and U. Bässler (1985) Physiology of the femoral chordotonal organ in the stick insect, Cuniculina impigra. J. Exp. Biol. 114: 207-223.

Hoyle, G., and M. Burrows (1973) Neural mechanisms underlying behavior in the locust Schistocerca gregaria. I. Physiology of identified motorneurons in the metathoracic ganglion. J. Neurobiol. 4: 341.

Kirk, M. D., and J. J. Wine (1984) Identified interneurons produce both primary afferent depolarization and presynaptic inhibition. Science 225: 854-856.

Macmillan, D. L., and J. Kien (1983) Intra- and intersegmental pathways active during walking in the locust. Proc. R. Soc. Lond. [Biol.] 218: 287-308.

Matthews, P. B. C. (1972) Mammalian Muscle Receptors and Their Central Actions, Arnold, London.

Pearson, K. G., R. K. S. Wong, and C. R. Fourtner (1976) Connexions between hair-plate afferents and motoneurones in the cockroach leg. J. Exp. Biol. 64: 251-266.

Pearson, K. G., W. J. Heitler, and J. D. Steeves (1980) Triggering of locust jump by multimodal inhibitory interneurons. J. Neurophysiol. 43: $257-278$

Pearson, K. G., D. N. Reye, and R. M. Robertson (1983) Phasedependent influences of wing stretch receptors on flight rhythm in the locust. J. Neurophysiol. 49: 1168-1181.

Phillips, C. E. (1981) Organization of motor neurons to a multiply innervated insect muscle. J. Neurobiol. 12: 269-280.

Pitman, R. M., C. D. Tweedle, and M. J. Cohen (1972) Branching of central neurons: Intracellular cobalt injection for light and electron microscopy. Science 176: 412-414.

Robertson, R. M., and K. G. Pearson (1985) Neural circuits in the flight system of the locust. I. Neurophysiol. 53: 1, 110-128.

Rudomin, P. (1980) Information processing at synapses in the ver- tebrate spinal cord: Presynaptic control of information transfer in monosynaptic pathways. In Information Processing in the Nervous System, H. M. Pinsker and W. D. Willis, eds., pp. 125-155, Raven, New York.

Selverston, A. I., H.-U. Kleindienst, and F. Huber (1985) Synaptic connectivity between cricket auditory interneurons as studied by selective photoinactivation. J. Neurosci. 5: 1283-1292.

Siegler, M. V. S., and M. Burrows (1983) Spiking local interneurons as primary integrators of mechanosensory information in the locust. J. Neurophysiol. 50: 1281-1295.

Siegler, M. V. S., and M. Burrows (1984) The morphology of two groups of spiking local interneurones in the metathoracic ganglion of the locust. J. Comp. Neurol. 224: 463-482.

Siegler, M. V. S., and M. Burrows (1986) Receptive fields of motor neurones underlying local tactile reflexes in the locust. J. Neurosci. 6: 507-513.

Sillar, T., and P. Skorupski (1986) Central input to primary afferent neurones in crayfish, Pacifastacus leniusculus, is correlated with rhythmic motor output of thoracic ganglia. J. Neurophysiol. 55: 678688.

Usherwood, P. N. R., H. I. Runion, and J. I. Campbell (1968) Structure and physiology of a chordotonal organ in the locust leg. J. Exp. Biol. 48: 305-323.

Watson, A. H. D., and M. Burrows (1987) Immunocytochemical and pharmacological evidence for GABAergic spiking local interneurons in the locust. J. Neurosci. (in press).

Watson, A. H. D., and H. J. Pflüger (1984) The ultrastructure of prosternal sensory hair afferents within the locust central nervous system. Neuroscience 11: 269-279.

Wine, J. J. (1977) Crayfish escape behaviour. III. Monosynaptic and polysynaptic sensory pathways involved in phasic extension. J. Comp. Physiol. 121: 187-203.

Zill, S. N. (1985a) Plasticity and proprioception in insects. I. Responses and cellular properties of individual receptors of the locust metathoracic femoral chordotonal organ. J. Exp. Biol. 116: 435-461.

Zill, S. N. (1985b) Plasticity and proprioception in insects. II. Modes of reflex action of the locust metathoracic femoral chordotonal organ. J. Exp. Biol. 116: 463-480. 